\title{
Some weighted integral inequalities for differentiable preinvex and prequasiinvex functions with applications
}

\author{
Muhammad Amer Latif ${ }^{*}$ and Sever Silvestru Dragomir ${ }^{1,2}$
}

${ }^{\text {} C}$ Correspondence:
m_amer_latif@hotmail.com
'School of Computational and
Applied Mathematics, University of
the Witwatersrand, Private Bag 3,
Wits 2050, Johannesburg, South
Africa
Full list of author information is
available at the end of the article

available at the end of the article

\begin{abstract}
In this paper, we present weighted integral inequalities of Hermite-Hadamard type for differentiable preinvex and prequasiinvex functions. Our results, on the one hand, give a weighted generalization of recent results for preinvex functions and, on the other hand, extend several results connected with the Hermite-Hadamard type integral inequalities. Applications of the obtained results are provided as well. MSC: 26D15; 26D20; 26D07

Keywords: Hermite-Hadamard's inequality; invex set; preinvex function; prequasiinvex; Hölder's integral inequality; power-mean inequality
\end{abstract}

\section{Introduction}

Let $f: I \subseteq \mathbb{R} \rightarrow \mathbb{R}$ be a convex mapping and $a, b \in I$ with $a<b$. Then

$$
f\left(\frac{a+b}{2}\right) \leq \frac{1}{b-a} \int_{a}^{b} f(x) d x \leq \frac{f(a)+f(b)}{2} .
$$

Both the inequalities in (1.1) hold in reversed direction if $f$ is concave. Inequalities (1.1) are famous in mathematical literature due to their rich geometrical significance and applications and are known as the Hermite-Hadamard inequalities (see [1]).

For several results which generalize, improve and extend inequalities (1.1), we refer the interested reader to [2-18].

In [3], Dragomir and Agarwal obtained the following inequalities for differentiable functions which estimate the difference between the middle and the rightmost terms in (1.1).

Theorem 1 [3] Let $f: I \subseteq \mathbb{R} \rightarrow \mathbb{R}$ be a differentiable mapping on $I^{\circ}$, where $a, b \in I$ with $a<b$, and $f^{\prime} \in L([a, b])$. If $\left|f^{\prime}\right|$ is a convex function on $[a, b]$, the following inequality holds:

$$
\left|\frac{f(a)+f(b)}{2}-\frac{1}{b-a} \int_{a}^{b} f(x) d x\right| \leq \frac{b-a}{8}\left[\left|f^{\prime}(a)\right|+\left|f^{\prime}(b)\right|\right] .
$$

Theorem 2 [3] Let $f: I \subseteq \mathbb{R} \rightarrow \mathbb{R}$ be a differentiable mapping on $I^{\circ}$, where $a, b \in I$ with $a<b$, and $f^{\prime} \in L([a, b])$. If $\left|f^{\prime}\right|^{\frac{p}{p-1}}$ is a convex function on $[a, b]$, the following inequality

\section{Springer}

@2013 Latif and Dragomir; licensee Springer. This is an Open Access article distributed under the terms of the Creative Commons Attribution License (http://creativecommons.org/licenses/by/2.0), which permits unrestricted use, distribution, and reproduction in any medium, provided the original work is properly cited. 
holds:

$$
\left|\frac{f(a)+f(b)}{2}-\frac{1}{b-a} \int_{a}^{b} f(x) d x\right| \leq \frac{b-a}{2(p+1)^{\frac{1}{p}}}\left[\left|f^{\prime}(a)\right|^{\frac{p}{p-1}}+\left|f^{\prime}(b)\right|^{\frac{p}{p-1}}\right],
$$

where $p>1$ and $\frac{1}{p}+\frac{1}{q}=1$.

In [11], Pearce and Pečarić gave an improvement and simplification of the constant in Theorem 2 and consolidated these results with Theorem 1 . The following is the main result from [11].

Theorem 3 [11] Let $f: I \subseteq \mathbb{R} \rightarrow \mathbb{R}$ be a differentiable mapping on $I^{\circ}$, where $a, b \in I$ with $a<b$, and $f^{\prime} \in L([a, b])$. If $\left|f^{\prime}\right|^{q}$ is a convex function on $[a, b]$, for some $q \geq 1$, the following inequality holds:

$$
\left|\frac{f(a)+f(b)}{2}-\frac{1}{b-a} \int_{a}^{b} f(x) d x\right| \leq \frac{b-a}{4}\left[\frac{\left|f^{\prime}(a)\right|^{q}+\left|f^{\prime}(b)\right|^{q}}{2}\right]^{\frac{1}{q}} .
$$

If $\left|f^{\prime}\right|^{q}$ is concave on $[a, b]$ for some $q \geq 1$, then

$$
\left|\frac{f(a)+f(b)}{2}-\frac{1}{b-a} \int_{a}^{b} f(x) d x\right| \leq \frac{b-a}{4}\left|f^{\prime}\left(\frac{a+b}{2}\right)\right| .
$$

Now, we recall that the notion of quasi-convex functions generalizes the notion of convex functions. More exactly, a function $f:[a, b] \rightarrow \mathbb{R}$ is said to be quasi-convex on $[a, b]$ if

$$
f(t x+(1-t) y) \leq \max \{f(x), f(y)\}
$$

for all $x, y \in[a, b]$ and $t \in[0,1]$. Clearly, any convex function is a quasi-convex function. Furthermore, there exist quasi-convex functions which are not convex (see [6]).

Recently, Ion [6] introduced two inequalities of the right-hand side of Hadamard type for quasi-convex functions, as follows.

Theorem 4 [6] Let $f: I \subseteq \mathbb{R} \rightarrow \mathbb{R}$ be a differentiable mapping on $I^{\circ}$, where $a, b \in I^{\circ}$ with $a<b$. If $\left|f^{\prime}\right|$ is a quasi-convex function on $[a, b]$, the following inequality holds:

$$
\left|\frac{f(a)+f(b)}{2}-\frac{1}{b-a} \int_{a}^{b} f(x) d x\right| \leq \frac{b-a}{4} \max \left\{\left|f^{\prime}(a)\right|,\left|f^{\prime}(b)\right|\right\} .
$$

Theorem 5 [6] Let $f: I \subseteq \mathbb{R} \rightarrow \mathbb{R}$ be a differentiable mapping on $I^{\circ}$, where $a, b \in I^{\circ}$ with $a<b$. If $\left|f^{\prime}\right|^{p}$ is a quasi-convex function on $[a, b]$, for some $p>1$, the following inequality holds:

$$
\begin{aligned}
& \left|\frac{f(a)+f(b)}{2}-\frac{1}{b-a} \int_{a}^{b} f(x) d x\right| \\
& \quad \leq \frac{b-a}{2(p+1)^{\frac{1}{p}}}\left[\max \left\{\left|f^{\prime}(a)\right|^{\frac{p}{p-1}},\left|f^{\prime}(b)\right|^{\frac{p}{p-1}}\right\}\right]^{\frac{p-1}{p}},
\end{aligned}
$$

where $\frac{1}{p}+\frac{1}{q}=1$. 
In [2], Alomari et al. established Hermite-Hadamard-type inequalities for quasi-convex functions which give refinements of those given above in Theorem 4 and Theorem 5 .

Theorem 6 [2] Let $f: I \subseteq[0, \infty) \rightarrow \mathbb{R}$ be a differentiable mapping on $I^{\circ}$ such that $f^{\prime} \in$ $L([a, b])$, where $a, b \in I^{\circ}$ with $a<b$. If the mapping $\left|f^{\prime}\right|$ is a quasi-convex function on $[a, b]$, the following inequality holds:

$$
\begin{aligned}
& \left|\frac{f(a)+f(b)}{2}-\frac{1}{b-a} \int_{a}^{b} f(x) d x\right| \\
& \quad \leq \frac{b-a}{8}\left[\max \left\{\left|f^{\prime}(a)\right|,\left|f^{\prime}\left(\frac{a+b}{2}\right)\right|\right\}+\max \left\{\left|f^{\prime}(b)\right|,\left|f^{\prime}\left(\frac{a+b}{2}\right)\right|\right\}\right] .
\end{aligned}
$$

Theorem 7 [2] Let $f: I \subseteq[0, \infty) \rightarrow \mathbb{R}$ be a differentiable mapping on $I^{\circ}$ such that $f^{\prime} \in$ $L([a, b])$, where $a, b \in I^{\circ}$ with $a<b$. If $\left|f^{\prime}\right|^{\frac{p}{p-1}}$ is a quasi-convex function on $[a, b]$, for $p>1$, the following inequality holds:

$$
\begin{aligned}
& \left|\frac{f(a)+f(b)}{2}-\frac{1}{b-a} \int_{a}^{b} f(x) d x\right| \\
& \leq \frac{b-a}{4(p+1)^{\frac{1}{p}}}\left[\left(\max \left\{\left|f^{\prime}(a)\right|^{\frac{p}{p-1}},\left|f^{\prime}\left(\frac{a+b}{2}\right)\right|^{\frac{p}{p-1}}\right\}\right)^{\frac{p-1}{p}}\right. \\
& \left.\quad+\left(\max \left\{\left|f^{\prime}(b)\right|^{\frac{p}{p-1}},\left|f^{\prime}\left(\frac{a+b}{2}\right)\right|^{\frac{p}{p-1}}\right\}\right)^{\frac{p-1}{p}}\right] .
\end{aligned}
$$

Theorem 8 [2] Let $f: I \subseteq[0, \infty) \rightarrow \mathbb{R}$ be a differentiable mapping on $I^{\circ}$ such that $f^{\prime} \in$ $L([a, b])$, where $a, b \in I^{\circ}$ with $a<b$. If $\left|f^{\prime}\right|^{q}$ is a quasi-convex function on $[a, b]$, for $q \geq 1$, the following inequality holds:

$$
\begin{aligned}
& \left|\frac{f(a)+f(b)}{2}-\frac{1}{b-a} \int_{a}^{b} f(x) d x\right| \\
& \leq \frac{b-a}{8}\left[\left(\max \left\{\left|f^{\prime}(a)\right|^{q},\left|f^{\prime}\left(\frac{a+b}{2}\right)\right|^{q}\right\}\right)^{\frac{1}{q}}\right. \\
& \left.\quad+\left(\max \left\{\left|f^{\prime}(b)\right|^{q},\left|f^{\prime}\left(\frac{a+b}{2}\right)\right|^{q}\right\}\right)^{\frac{1}{q}}\right] .
\end{aligned}
$$

In [5], Hwang established the following results for convex and quasi-convex functions; those results provide a weighted generalization of the results given in Theorem 1, Theorem 3, Theorem 6 and Theorem 8.

Theorem 9 [5] Let $f: I \subseteq \mathbb{R} \rightarrow \mathbb{R}$ be a differentiable mapping on $I^{\circ}$, where $a, b \in I^{\circ}$ with $a<b$, and let $g:[a, b] \rightarrow[0, \infty)$ be a continuous positive mapping and symmetric to $\frac{a+b}{2}$. If $\left|f^{\prime}\right|$ is a convex function on $[a, b]$, the following inequality holds:

$$
\begin{aligned}
& \left|\left[\frac{f(a)+f(b)}{2}\right] \int_{a}^{b} g(x) d x-\int_{a}^{b} f(x) g(x) d x\right| \\
& \quad \leq \frac{b-a}{4}\left[\left|f^{\prime}(a)\right|+\left|f^{\prime}(b)\right|\right] \int_{0}^{1} \int_{L(a, b, t)}^{U(a, b, t)} g(x) d x d t,
\end{aligned}
$$

where $U(a, b, t)=\frac{1-t}{2} a+\frac{1+t}{2} b$ and $L(a, b, t)=\frac{1+t}{2} a+\frac{1-t}{2} b$. 
Theorem 10 [5] Suppose that the assumptions of Theorem 9 are satisfied and $q \geq 1$. If $\left|f^{\prime}\right|^{q}$ is a convex function on $[a, b]$, the following inequality holds:

$$
\begin{aligned}
& \left|\left[\frac{f(a)+f(b)}{2}\right] \int_{a}^{b} g(x) d x-\int_{a}^{b} f(x) g(x) d x\right| \\
& \quad \leq \frac{b-a}{2}\left[\frac{\left|f^{\prime}(a)\right|^{q}+\left|f^{\prime}(b)\right|^{q}}{2}\right]^{\frac{1}{q}} \int_{0}^{1} \int_{L(a, b, t)}^{U(a, b, t)} g(x) d x d t,
\end{aligned}
$$

where $U(a, b, t)$ and $L(a, b, t)$ are as defined in Theorem 9.

Theorem 11 [5] Suppose that the assumptions of Theorem 9 are satisfied. If $\left|f^{\prime}\right|$ is a quasiconvex function on $[a, b]$, the following inequality holds:

$$
\begin{aligned}
& \left|\left[\frac{f(a)+f(b)}{2}\right] \int_{a}^{b} g(x) d x-\int_{a}^{b} f(x) g(x) d x\right| \\
& \leq \frac{b-a}{4}\left[\max \left\{\left|f^{\prime}(a)\right|,\left|f^{\prime}\left(\frac{a+b}{2}\right)\right|\right\}+\max \left\{\left|f^{\prime}(b)\right|,\left|f^{\prime}\left(\frac{a+b}{2}\right)\right|\right\}\right] \\
& \quad \times \int_{0}^{1} \int_{L(a, b, t)}^{U(a, b, t)} g(x) d x d t,
\end{aligned}
$$

where $U(a, b, t)$ and $L(a, b, t)$ are as defined in Theorem 9.

Theorem 12 [5] Suppose that the assumptions of Theorem 9 are satisfied and $q \geq 1$. If $\left|f^{\prime}\right|^{q}$ is a quasi-convex function on $[a, b]$, the following inequality holds:

$$
\begin{aligned}
& \left|\left[\frac{f(a)+f(b)}{2}\right] \int_{a}^{b} g(x) d x-\int_{a}^{b} f(x) g(x) d x\right| \\
& \quad \leq \frac{b-a}{4}\left[\left(\max \left\{\left|f^{\prime}(a)\right|^{q},\left|f^{\prime}\left(\frac{a+b}{2}\right)\right|^{q}\right\}\right)^{\frac{1}{q}}\right. \\
& \left.\quad+\left(\max \left\{\left|f^{\prime}(b)\right|^{q},\left|f^{\prime}\left(\frac{a+b}{2}\right)\right|^{q}\right\}\right)^{\frac{1}{q}}\right] \int_{0}^{1} \int_{L(a, b, t)}^{U(a, b, t)} g(x) d x d t,
\end{aligned}
$$

where $U(a, b, t)$ and $L(a, b, t)$ are as defined in Theorem 9.

In recent years, a lot of efforts have been made by many mathematicians to generalize the classical convexity. These studies include, among others, the work of Hanson [19], BenIsrael and Mond [20], Pini [21], Noor [22, 23], Yang and Li [24] and Weir and Mond [25]. Ben-Israel and Mond [20], Weir and Mond [25] and Noor [22, 23] have studied the basic properties of the preinvex functions and their role in optimization, variational inequalities and equilibrium problems. Hanson [19] introduced invex functions as a significant generalization of the convex functions. Ben-Israel and Mond [20] gave the concept of preinvex functions which is a special case of invexity. Pini [21] introduced the concept of prequasiinvex functions as a generalization of invex functions.

Let us recall some known results concerning preinvexity and prequasiinvexity.

Let $K$ be a subset in $\mathbb{R}^{n}$ and let $f: K \rightarrow \mathbb{R}$ and $\eta: K \times K \rightarrow \mathbb{R}^{n}$ be continuous functions. Let $x \in K$, then the set $K$ is said to be invex at $x$ with respect to $\eta(\cdot, \cdot)$ if

$$
x+t \eta(y, x) \in K, \quad \forall x, y \in K, t \in[0,1] .
$$


$K$ is said to be an invex set with respect to $\eta$ if $K$ is invex at each $x \in K$. The invex set $K$ is also called an $\eta$-connected set.

Definition 1 [25] The function $f$ on the invex set $K$ is said to be preinvex with respect to $\eta$ if

$$
f(u+t \eta(v, u)) \leq(1-t) f(u)+t f(v), \quad \forall u, v \in K, t \in[0,1] .
$$

The function $f$ is said to be preconcave if and only if $-f$ is preinvex.

It is to be noted that every convex function is preinvex with respect to the map $\eta(x, y)=$ $x-y$, but the converse is not true; see, for instance, [18].

Definition 2 [26] The function $f$ on the invex set $K$ is said to be prequasiinvex with respect to $\eta$ if

$$
f(u+t \eta(v, u)) \leq \max \{f(u), f(v)\}, \quad \forall u, v \in K, t \in[0,1] .
$$

Also every quasi-convex function is prequasiinvex with respect to the map $\eta(v, u)=v-u$, but the converse does not hold; see, for example, [27].

In the recent paper, Noor [28] obtained the following Hermite-Hadamard inequalities for the preinvex functions.

Theorem 13 [28] Let $f:[a, a+\eta(b, a)] \rightarrow(0, \infty)$ be a preinvex function on the interval of the real numbers $K^{\circ}$ (the interior of $K$ ) and $a, b \in K^{\circ}$ with $\eta(b, a)>0$. Then the following inequalities hold:

$$
f\left(\frac{2 a+\eta(b, a)}{2}\right) \leq \frac{1}{\eta(b, a)} \int_{a}^{a+\eta(b, a)} f(x) d x \leq \frac{f(a)+f(b)}{2} .
$$

Barani et al. in [29] presented the following estimates of the right-hand side of a Hermite-Hadamard-type inequality in which some preinvex functions are involved.

Theorem 14 [29] Let $K \subseteq \mathbb{R}$ be an open invex subset with respect to $\eta: K \times K \rightarrow \mathbb{R}$. Suppose that $f: K \rightarrow \mathbb{R}$ is a differentiable function. If $\left|f^{\prime}\right|$ is preinvex on $K$, for every $a, b \in K$ with $\eta(b, a) \neq 0$, the following inequality holds:

$$
\left|\frac{f(a)+f(a+\eta(b, a))}{2}-\frac{1}{\eta(b, a)} \int_{a}^{a+\eta(b, a)} f(x) d x\right| \leq \frac{|\eta(b, a)|}{8}\left(\left|f^{\prime}(a)\right|+\left|f^{\prime}(b)\right|\right) .
$$

Theorem 15 [29] Let $K \subseteq \mathbb{R}$ be an open invex subset with respect to $\eta: K \times K \rightarrow \mathbb{R}$. Suppose that $f: K \rightarrow \mathbb{R}$ is a differentiable function. Assume $p \in \mathbb{R}$ with $p>1$. If $\left|f^{\prime}\right|^{\frac{p}{p-1}}$ is preinvex on $K$, for every $a, b \in K$ with $\eta(b, a) \neq 0$, the following inequality holds:

$$
\begin{gathered}
\left|\frac{f(a)+f(a+\eta(b, a))}{2}-\frac{1}{\eta(b, a)} \int_{a}^{a+\eta(b, a)} f(x) d x\right| \\
\leq \frac{|\eta(b, a)|}{2(1+p)^{\frac{1}{p}}}\left[\frac{\left|f^{\prime}(a)\right|^{\frac{p}{p-1}}+\left|f^{\prime}(b)\right|^{\frac{p}{p-1}}}{2}\right]^{\frac{p-1}{p}} .
\end{gathered}
$$


In [30], Barani et al. gave similar results for prequasiinvex functions as follows.

Theorem 16 [30] Let $K \subseteq \mathbb{R}$ be an open invex subset with respect to $\eta: K \times K \rightarrow \mathbb{R}$. Suppose that $f: K \rightarrow \mathbb{R}$ is a differentiable function. If $\left|f^{\prime}\right|$ is prequasiinvex on $K$, for every $a, b \in K$ with $\eta(b, a) \neq 0$, the following inequality holds:

$$
\begin{aligned}
& \left|\frac{f(a)+f(a+\eta(b, a))}{2}-\frac{1}{\eta(b, a)} \int_{a}^{a+\eta(b, a)} f(x) d x\right| \\
& \quad \leq \frac{|\eta(b, a)|}{8} \max \left\{\left|f^{\prime}(a)\right|,\left|f^{\prime}(b)\right|\right\} .
\end{aligned}
$$

Theorem 17 [30] Let $K \subseteq \mathbb{R}$ be an open invex subset with respect to $\eta: K \times K \rightarrow \mathbb{R}$. Suppose that $f: K \rightarrow \mathbb{R}$ is a differentiable function. Assume $p \in \mathbb{R}$ with $p>1$. If $\left|f^{\prime}\right|^{\frac{p}{p-1}}$ is prequasiinvex on $K$, for every $a, b \in K$ with $\eta(b, a) \neq 0$, the following inequality holds:

$$
\begin{gathered}
\left|\frac{f(a)+f(a+\eta(b, a))}{2}-\frac{1}{\eta(b, a)} \int_{a}^{a+\eta(b, a)} f(x) d x\right| \\
\quad \leq \frac{|\eta(b, a)|}{2(1+p)^{\frac{1}{p}}}\left(\max \left\{\left|f^{\prime}(a)\right|^{\frac{p}{p-1}},\left|f^{\prime}(b)\right|^{\frac{p}{p-1}}\right\}\right)^{\frac{p-1}{p}} .
\end{gathered}
$$

Latif [31] proved the following results which give a refinement of the results given in Theorems 14-17.

Theorem 18 [31] Let $K \subseteq[0, \infty)$ be an open invex subset with respect to $\eta: K \times K \rightarrow \mathbb{R}$. Suppose that $f: K \rightarrow \mathbb{R}$ is a differentiable mapping on $K$ such that $f^{\prime} \in L([a, a+\eta(b, a)])$. If $\left|f^{\prime}\right|$ is prequasiinvex on $K$, then for every $a, b \in K$ with $\eta(b, a)>0$, we have the following inequality:

$$
\begin{aligned}
& \left|\frac{f(a)+f(a+\eta(b, a))}{2}-\frac{1}{\eta(b, a)} \int_{a}^{a+\eta(b, a)} f(x) d x\right| \\
& \leq \frac{\eta(b, a)}{8}\left[\max \left\{\left|f^{\prime}(a)\right|,\left|f^{\prime}\left(a+\frac{1}{2} \eta(b, a)\right)\right|\right\}\right. \\
& \left.\quad+\max \left\{\left|f^{\prime}\left(a+\frac{1}{2} \eta(b, a)\right)\right|,\left|f^{\prime}(a+\eta(b, a))\right|\right\}\right] .
\end{aligned}
$$

Theorem 19 [31] Let $K \subseteq[0, \infty)$ be an open invex subset with respect to $\eta: K \times K \rightarrow \mathbb{R}$. Suppose that $f: K \rightarrow \mathbb{R}$ is a differentiable mapping on $K$ such that $f^{\prime} \in L([a, a+\eta(b, a)])$. If $\left|f^{\prime}\right|^{p}$ is prequasiinvex on $K$ for some $p>1$, then for every $a, b \in K$ with $\eta(b, a)>0$, we have the following inequality:

$$
\begin{aligned}
& \left|\frac{f(a)+f(a+\eta(b, a))}{2}-\frac{1}{\eta(b, a)} \int_{a}^{a+\eta(b, a)} f(x) d x\right| \\
& \leq \frac{\eta(b, a)}{4(p+1)^{\frac{1}{p}}}\left[\left(\max \left\{\left|f^{\prime}(a)\right|^{\frac{p}{p-1}},\left|f^{\prime}\left(a+\frac{1}{2} \eta(b, a)\right)\right|^{\frac{p}{p-1}}\right\}\right)^{\frac{p-1}{p}}\right. \\
& \left.\quad+\left(\max \left\{\left|f^{\prime}\left(a+\frac{1}{2} \eta(b, a)\right)\right|^{\frac{p}{p-1}},\left|f^{\prime}(a+\eta(b, a))\right|^{\frac{p}{p-1}}\right\}\right)^{\frac{p-1}{p}}\right] .
\end{aligned}
$$


Theorem 20 [31] Let $K \subseteq \mathbb{R}$ be an open invex subset with respect to $\eta: K \times K \rightarrow \mathbb{R}$. Suppose that $f: K \rightarrow \mathbb{R}$ is a differentiable mapping on $K$ such that $f^{\prime} \in L([a, a+\eta(b, a)])$. If $\left|f^{\prime}\right|^{q}$ for $q \geq 1$ is prequasiinvex on $K$, then for every $a, b \in K$ with $\eta(b, a)>0$, we have the following inequality:

$$
\begin{aligned}
& \left|\frac{f(a)+f(a+\eta(b, a))}{2}-\frac{1}{\eta(b, a)} \int_{a}^{a+\eta(b, a)} f(x) d x\right| \\
& \leq \frac{\eta(b, a)}{8}\left[\left(\max \left\{\left|f^{\prime}(a)\right|^{q},\left|f^{\prime}\left(a+\frac{1}{2} \eta(b, a)\right)\right|^{q}\right\}\right)^{\frac{1}{q}}\right. \\
& \left.\quad+\left(\max \left\{\left|f^{\prime}\left(a+\frac{1}{2} \eta(b, a)\right)\right|^{q},\left|f^{\prime}(a+\eta(b, a))\right|^{q}\right\}\right)^{\frac{1}{q}}\right] .
\end{aligned}
$$

For several new results on inequalities for preinvex and prequasiinvex functions, we refer the interested reader to $[26,29,32]$ and the references therein.

In the present paper we give new inequalities of Hermite-Hadamard for functions whose derivatives in absolute value are preinvex and prequasiinvex. Our results extend those results presented in very recent results from $[2,3,5,6]$ and [12] and generalize those results from $[29,30]$ and [33].

\section{Main results}

The following lemma is essential in establishing our main results in this section.

Lemma 1 Let $K \subseteq \mathbb{R}$ be an open invex subset with respect to $\eta: K \times K \rightarrow \mathbb{R}$ and $a, b \in$ $K$ with $\eta(b, a)>0$. Suppose that $f: K \rightarrow \mathbb{R}$ is a differentiable mapping on $K$ such that $f^{\prime} \in L([a, a+\eta(b, a)])$. If $h:[a, a+\eta(b, a)] \rightarrow[0, \infty)$ is a differentiable mapping, then the following equality holds:

$$
\begin{aligned}
& \frac{1}{2}[(h(a+\eta(b, a))-2 h(a)) f(a)+h(a+\eta(b, a)) f(a+\eta(b, a))]-\int_{a}^{a+\eta(b, a)} f(x) h^{\prime}(x) d x \\
& =\frac{\eta(b, a)}{4}\left\{\int_{0}^{1}\left[2 h\left(a+\left(\frac{1-t}{2}\right) \eta(b, a)\right)-h(a+\eta(b, a))\right] f^{\prime}\left(a+\left(\frac{1-t}{2}\right) \eta(b, a)\right) d t\right. \\
& \quad+\int_{0}^{1}\left[2 h\left(a+\left(\frac{1+t}{2}\right) \eta(b, a)\right)-h(a+\eta(b, a))\right] \\
& \left.\quad \times f^{\prime}\left(a+\left(\frac{1+t}{2}\right) \eta(b, a)\right) d t\right\} .
\end{aligned}
$$

Proof It suffices to note that

$$
\begin{aligned}
I_{1}= & \int_{0}^{1}\left[2 h\left(a+\left(\frac{1-t}{2}\right) \eta(b, a)\right)-h(a+\eta(b, a))\right] f^{\prime}\left(a+\left(\frac{1-t}{2}\right) \eta(b, a)\right) d t \\
= & -\left.2 \frac{\left[2 h\left(a+\left(\frac{1-t}{2}\right) \eta(b, a)\right)-h(a+\eta(b, a))\right] f\left(a+\left(\frac{1-t}{2}\right) \eta(b, a)\right)}{\eta(b, a)}\right|_{0} ^{1} \\
& -2 \int_{0}^{1} h^{\prime}\left(a+\left(\frac{1-t}{2}\right) \eta(b, a)\right) f\left(a+\left(\frac{1-t}{2}\right) \eta(b, a)\right) d t \\
= & \frac{-2[2 h(a)-h(a+\eta(b, a))] f(a)}{\eta(b, a)}
\end{aligned}
$$




$$
\begin{aligned}
& +\frac{2\left[2 h\left(a+\frac{1}{2} \eta(b, a)\right)-h(a+\eta(b, a))\right] f\left(a+\frac{1}{2} \eta(b, a)\right)}{\eta(b, a)} \\
& -2 \int_{0}^{1} h^{\prime}\left(a+\left(\frac{1-t}{2}\right) \eta(b, a)\right) f\left(a+\left(\frac{1-t}{2}\right) \eta(b, a)\right) d t
\end{aligned}
$$

Setting $x=a+\left(\frac{1-t}{2}\right) \eta(b, a)$ and $d x=-\frac{\eta(b, a)}{2} d t$, which gives

$$
\begin{aligned}
I_{1}= & \frac{2[h(a+\eta(b, a))-2 h(a)] f(a)}{\eta(b, a)}-\frac{4}{\eta(b, a)} \int_{a}^{a+\frac{1}{2} \eta(b, a)} h^{\prime}(x) f(x) d x \\
& +\frac{2\left[2 h\left(a+\frac{1}{2} \eta(b, a)\right)-h(a+\eta(b, a))\right] f\left(a+\frac{1}{2} \eta(b, a)\right)}{\eta(b, a)} .
\end{aligned}
$$

Similarly, we also have

$$
\begin{aligned}
I_{2}= & \int_{0}^{1}\left[2 h\left(a+\left(\frac{1+t}{2}\right) \eta(b, a)\right)-h(a+\eta(b, a))\right] f^{\prime}\left(a+\left(\frac{1+t}{2}\right) \eta(b, a)\right) d t \\
= & \frac{2 h(a+\eta(b, a)) f(a+\eta(b, a))}{\eta(b, a)}-\frac{4}{\eta(b, a)} \int_{a+\frac{1}{2} \eta(b, a)}^{a+\eta(b, a)} h^{\prime}(x) f(x) d x \\
& -\frac{2\left[2 h\left(a+\frac{1}{2} \eta(b, a)\right)-h(a+\eta(b, a))\right] f\left(a+\frac{1}{2} \eta(b, a)\right)}{\eta(b, a)} .
\end{aligned}
$$

Thus, from (2.2) and (2.3), we have

$$
\begin{aligned}
\frac{\eta(b, a)}{4}\left[I_{1}+I_{2}\right]= & \frac{1}{2}[(h(a+\eta(b, a))-2 h(a)) f(a)+h(a+\eta(b, a)) f(a+\eta(b, a))] \\
& -\int_{a}^{a+\eta(b, a)} f(x) h^{\prime}(x) d x
\end{aligned}
$$

which is the required result.

Remark 1 If we take $\eta(b, a)=b-a$, then Lemma 1 reduces to Lemma 2.1 from [5].

Now using Lemma 1, we shall propose some new upper bounds for the difference between the rightmost and middle terms of a weighted version of the Hadamard inequality (1.15) using preinvex and prequasiinvex mappings. Our results provide a weighted generalization of those results given in $[29,30]$ and [31].

In what follows we use the notations $L^{\prime}(a, b, t)=a+\left(\frac{1-t}{2}\right) \eta(b, a)$ and $U^{\prime}(a, b, t)=a+$ $\left(\frac{1+t}{2}\right) \eta(b, a)$.

Theorem 21 Let $K \subseteq \mathbb{R}$ be an open invex subset with respect to $\eta: K \times K \rightarrow \mathbb{R}$ and $a, b \in K$ with $\eta(b, a)>0$. Suppose that $f: K \rightarrow \mathbb{R}$ is a differentiable mapping on $K$ and $w:[a, a+$ $\eta(b, a)] \rightarrow[0, \infty)$ is continuous and symmetric to $a+\frac{1}{2} \eta(b, a)$. If $\left|f^{\prime}\right|$ is preinvex on $K$, we have the following inequality:

$$
\begin{aligned}
& \left|\frac{f(a)+f(a+\eta(b, a))}{2} \int_{a}^{a+\eta(b, a)} w(x) d x-\int_{a}^{a+\eta(b, a)} f(x) w(x) d x\right| \\
& \quad \leq \frac{\eta(b, a)}{4}\left[\left|f^{\prime}(a)\right|+\left|f^{\prime}(b)\right|\right] \int_{0}^{1} \int_{L^{\prime}(a, b, t)}^{U^{\prime}(a, b, t)} w(x) d x d t .
\end{aligned}
$$


Proof Let $h(t)=\int_{a}^{t} w(t) d t$ for all $t \in[a, a+\eta(b, a)]$ in Lemma 1, we obtain

$$
\begin{aligned}
\mid \frac{f(a)}{}+f(a+\eta(b, a)) & \int_{a}^{a+\eta(b, a)} w(t) d t-\int_{a}^{a+\eta(b, a)} f(x) w(x) d x \mid \\
\leq & \frac{\eta(b, a)}{4}\left\{\int_{0}^{1}\left|2 h\left(a+\left(\frac{1-t}{2}\right) \eta(b, a)\right)-h(a+\eta(b, a))\right|\right. \\
\quad & \left|f^{\prime}\left(a+\left(\frac{1-t}{2}\right) \eta(b, a)\right)\right| d t \\
& +\int_{0}^{1}\left|2 h\left(a+\left(\frac{1+t}{2}\right) \eta(b, a)\right)-h(a+\eta(b, a))\right| \\
& \left.\times\left|f^{\prime}\left(a+\left(\frac{1+t}{2}\right) \eta(b, a)\right)\right| d t\right\} .
\end{aligned}
$$

Since $w(x)$ is symmetric to $a+\frac{1}{2} \eta(b, a)$, we have

$$
\left|2 h\left(a+\left(\frac{1-t}{2}\right) \eta(b, a)\right)-h(a+\eta(b, a))\right|=\int_{L^{\prime}(a, b, t)}^{U^{\prime}(a, b, t)} w(x) d x
$$

and

$$
\left|2 h\left(a+\left(\frac{1+t}{2}\right) \eta(b, a)\right)-h(a+\eta(b, a))\right|=\int_{L^{\prime}(a, b, t)}^{u^{\prime}(a, b, t)} w(x) d x
$$

for all $t \in[0,1]$. Using (2.6) and (2.7) in (2.5), we have

$$
\begin{aligned}
& \left|\frac{f(a)+f(a+\eta(b, a))}{2} \int_{a}^{a+\eta(b, a)} w(t) d t-\int_{a}^{a+\eta(b, a)} f(x) w(x) d x\right| \\
& \leq \frac{\eta(b, a)}{4} \int_{0}^{1}\left(\int_{L^{\prime}(a, b, t)}^{U^{\prime}(a, b, t)} w(x) d x\right)\left[\left|f^{\prime}\left(a+\left(\frac{1-t}{2}\right) \eta(b, a)\right)\right|\right. \\
& \left.\quad+\left|f^{\prime}\left(a+\left(\frac{1+t}{2}\right) \eta(b, a)\right)\right|\right] d t .
\end{aligned}
$$

Since $\left|f^{\prime}\right|$ is preinvex on $K$, hence for every $a, b \in K$ with $\eta(b, a)>0$, we have

$$
\begin{aligned}
& \left|f^{\prime}\left(a+\left(\frac{1-t}{2}\right) \eta(b, a)\right)\right|+\left|f^{\prime}\left(a+\left(\frac{1+t}{2}\right) \eta(b, a)\right)\right| \\
& \quad \leq\left(\frac{1+t}{2}\right)\left|f^{\prime}(a)\right|+\left(\frac{1-t}{2}\right)\left|f^{\prime}(b)\right|+\left(\frac{1-t}{2}\right)\left|f^{\prime}(a)\right|+\left(\frac{1+t}{2}\right)\left|f^{\prime}(b)\right| \\
& \quad=\left|f^{\prime}(a)\right|+\left|f^{\prime}(b)\right| .
\end{aligned}
$$

Using (2.9) in (2.8), we get the required inequality. This completes the proof of the theorem.

Remark 2 In Theorem 21, if we take $w(x)=\frac{1}{\eta(b, a)}$ for all $x \in[a, a+\eta(b, a)]$, then (2.4) becomes inequality (1.16).

Remark 3 If $\eta(b, a)=b-a$ in Theorem 21, then (2.4) reduces to inequality (1.11) from [5]. 
Theorem 22 Let $K \subseteq \mathbb{R}$ be an open invex subset with respect to $\eta: K \times K \rightarrow \mathbb{R}$ and $a, b \in K$ with $\eta(b, a)>0$. Suppose that $f: K \rightarrow \mathbb{R}$ is a differentiable mapping on $K$ and $w:[a, a+$ $\eta(b, a)] \rightarrow[0, \infty)$ is continuous and symmetric to $a+\frac{1}{2} \eta(b, a)$. If $\left|f^{\prime}\right|^{q}$ is preinvex on $K$ for $q>1$, we have the following inequality:

$$
\begin{aligned}
& \left|\frac{f(a)+f(a+\eta(b, a))}{2} \int_{a}^{a+\eta(b, a)} w(x) d x-\int_{a}^{a+\eta(b, a)} f(x) w(x) d x\right| \\
& \quad \leq \frac{\eta(b, a)}{2}\left[\frac{\left|f^{\prime}(a)\right|^{q}+\left|f^{\prime}(b)\right|^{q}}{2}\right]^{\frac{1}{q}}\left(\int_{0}^{1}\left[\int_{L^{\prime}(a, b, t)}^{u^{\prime}(a, b, t)} w(x) d x\right]^{p} d t\right)^{\frac{1}{p}},
\end{aligned}
$$

where $\frac{1}{p}+\frac{1}{q}=1$.

Proof Continuing from inequality (2.8) in the proof of Theorem 21 and using the wellknown Hölder integral inequality, we have

$$
\begin{gathered}
\left|\frac{f(a)+f(a+\eta(b, a))}{2} \int_{a}^{a+\eta(b, a)} w(t) d t-\int_{a}^{a+\eta(b, a)} f(x) w(x) d x\right| \\
\leq \frac{\eta(b, a)}{4}\left(\int_{0}^{1}\left[\int_{L^{\prime}(a, b, t)}^{U^{\prime}(a, b, t)} w(x) d x\right]^{p} d t\right)^{\frac{1}{p}} \\
\times\left[\left(\int_{0}^{1}\left|f^{\prime}\left(a+\left(\frac{1-t}{2}\right) \eta(b, a)\right)\right|^{q} d t\right)^{\frac{1}{q}}\right. \\
\left.+\left(\int_{0}^{1}\left|f^{\prime}\left(a+\left(\frac{1+t}{2}\right) \eta(b, a)\right)\right|^{q} d t\right)^{\frac{1}{q}}\right] .
\end{gathered}
$$

By the power-mean inequality $t^{r}+s^{r}<2^{1-r}(t+s)^{r}$ for $t>0, s>0$ and $r<1$, and by the preinvexity of $\left|f^{\prime}\right|^{q}$ on $K$ for $q>1$, we have, for every $a, b \in K$ with $\eta(b, a)>0$, the following inequality:

$$
\begin{aligned}
& \left(\int_{0}^{1}\left|f^{\prime}\left(a+\left(\frac{1-t}{2}\right) \eta(b, a)\right)\right|^{q} d t\right)^{\frac{1}{q}}+\left(\int_{0}^{1}\left|f^{\prime}\left(a+\left(\frac{1+t}{2}\right) \eta(b, a)\right)\right|^{q} d t\right)^{\frac{1}{q}} \\
& \leq 2^{1-\frac{1}{q}}\left[\int_{0}^{1}\left|f^{\prime}\left(a+\left(\frac{1-t}{2}\right) \eta(b, a)\right)\right|^{q} d t+\int_{0}^{1}\left|f^{\prime}\left(a+\left(\frac{1+t}{2}\right) \eta(b, a)\right)\right|^{q} d t\right]^{\frac{1}{q}} \\
& \leq 2^{1-\frac{1}{q}}\left[\int _ { 0 } ^ { 1 } \left\{\left(\frac{1+t}{2}\right)\left|f^{\prime}(a)\right|^{q}+\left(\frac{1-t}{2}\right)\left|f^{\prime}(b)\right|^{q}\right.\right. \\
& \left.\left.\quad+\left(\frac{1-t}{2}\right)\left|f^{\prime}(a)\right|^{q}+\left(\frac{1+t}{2}\right)\left|f^{\prime}(b)\right|^{q}\right\} d t\right]^{\frac{1}{q}} \\
& =2^{1-\frac{1}{q}}\left[\left|f^{\prime}(a)\right|^{q}+\left|f^{\prime}(b)\right|^{q}\right]^{\frac{1}{q}} .
\end{aligned}
$$

Using the last inequality (2.12) in (2.11), we get the desired inequality. This completes the proof of the theorem as well.

Remark 4 In Theorem 22 if we take $w(x)=\frac{1}{\eta(b, a)}$ for all $x \in[a, a+\eta(b, a)]$ with $\eta(b, a)>0$, then (2.10) reduces to inequality (1.17). 
Remark 5 If we take $\eta(b, a)=b-a$ in Theorem 22, then (2.10) reduces to the following inequality:

$$
\begin{aligned}
& \left|\frac{f(a)+f(b)}{2} \int_{a}^{b} w(x) d x-\int_{a}^{b} f(x) w(x) d x\right| \\
& \quad \leq \frac{b-a}{2}\left[\frac{\left|f^{\prime}(a)\right|^{q}+\left|f^{\prime}(b)\right|^{q}}{2}\right]^{\frac{1}{q}}\left(\int_{0}^{1}\left[\int_{L(a, b, t)}^{U(a, b, t)} w(x) d x\right]^{p} d t\right)^{\frac{1}{p}},
\end{aligned}
$$

where $\frac{1}{p}+\frac{1}{q}=1, L(a, b, t)=\left(\frac{1+t}{2}\right) a+\left(\frac{1-t}{2}\right) b, U(a, b, t)=\left(\frac{1-t}{2}\right) a+\left(\frac{1+t}{2}\right) b, t \in[a, b]$.

A similar result may be stated as follows.

Theorem 23 Let $K \subseteq \mathbb{R}$ be an open invex subset with respect to $\eta: K \times K \rightarrow \mathbb{R}$. Suppose that $f: K \rightarrow \mathbb{R}$ is a differentiable mapping on $K$ and $w:[a, a+\eta(b, a)] \rightarrow[0, \infty)$ is continuous and symmetric to $a+\frac{1}{2} \eta(b, a)$. If $\left|f^{\prime}\right|^{q}$ is preinvex on $K$ for $q \geq 1$, then for every $a, b \in K$ with $\eta(b, a)>0$, we have the following inequality:

$$
\begin{gathered}
\left|\frac{f(a)+f(a+\eta(b, a))}{2} \int_{a}^{a+\eta(b, a)} w(x) d x-\int_{a}^{a+\eta(b, a)} f(x) w(x) d x\right| \\
\leq \frac{\eta(b, a)}{2}\left[\frac{\left|f^{\prime}(a)\right|^{q}+\left|f^{\prime}(b)\right|^{q}}{2}\right]^{\frac{1}{q}} \int_{0}^{1} \int_{L^{\prime}(a, b, t)}^{U^{\prime}(a, b, t)} w(x) d x d t .
\end{gathered}
$$

Proof Continuing from inequality (2.8) in the proof of Theorem 21 and using the wellknown Hölder integral inequality, we have

$$
\begin{aligned}
& \left|\frac{f(a)+f(a+\eta(b, a))}{2} \int_{a}^{a+\eta(b, a)} w(t) d t-\int_{a}^{a+\eta(b, a)} f(x) w(x) d x\right| \\
& \leq \frac{\eta(b, a)}{4}\left[\int_{0}^{1}\left(\int_{L^{\prime}(a, b, t)}^{U^{\prime}(a, b, t)} w(x) d x\right) d t\right]^{1-\frac{1}{q}} \\
& \times\left[\left\{\left(\int_{0}^{1}\left(\int_{L^{\prime}(a, b, t)}^{U^{\prime}(a, b, t)} w(x) d x\right)\left|f^{\prime}\left(a+\left(\frac{1-t}{2}\right) \eta(b, a)\right)\right|^{q} d t\right)\right\}^{\frac{1}{q}}\right. \\
& \left.+\left\{\left(\int_{0}^{1}\left(\int_{L^{\prime}(a, b, t)}^{U^{\prime}(a, b, t)} w(x) d x\right)\left|f^{\prime}\left(a+\left(\frac{1+t}{2}\right) \eta(b, a)\right)\right|^{q} d t\right)\right\}^{\frac{1}{q}}\right] .
\end{aligned}
$$

By the power-mean inequality $t^{r}+s^{r}<2^{1-r}(t+s)^{r}$ for $t>0, s>0$ and $r<1$, and by the preinvexity of $\left|f^{\prime}\right|^{q}$ on $K$ for $q>1$, we have, for every $a, b \in K$ with $\eta(b, a)>0$, the following inequality:

$$
\begin{aligned}
& \left\{\left(\int_{0}^{1}\left(\int_{L^{\prime}(a, b, t)}^{U^{\prime}(a, b, t)} w(x) d x\right)\left|f^{\prime}\left(a+\left(\frac{1-t}{2}\right) \eta(b, a)\right)\right|^{q} d t\right)\right\}^{\frac{1}{q}} \\
& +\left\{\left(\int_{0}^{1}\left(\int_{L^{\prime}(a, b, t)}^{U^{\prime}(a, b, t)} w(x) d x\right)\left|f^{\prime}\left(a+\left(\frac{1+t}{2}\right) \eta(b, a)\right)\right|^{q} d t\right)\right\}^{\frac{1}{q}} \\
& \leq 2^{1-\frac{1}{q}}\left[\int_{0}^{1}\left(\int_{L^{\prime}(a, b, t)}^{U^{\prime}(a, b, t)} w(x) d x\right) d t\right]^{\frac{1}{q}}\left[\left|f^{\prime}(a)\right|^{q}+\left|f^{\prime}(b)\right|^{q}\right]^{\frac{1}{q}} .
\end{aligned}
$$


Utilizing inequality (2.16) in (2.15), we get inequality (2.14). This completes the proof of the theorem.

Corollary 1 Suppose that all the assumptions of Theorem 23 are satisfied and if $w(x)=$ $\frac{1}{\eta(b, a)}$ for all $x \in[a, a+\eta(b, a)]$ with $\eta(b, a)>0$, then we have the following inequality:

$$
\begin{aligned}
& \left|\frac{f(a)+f(a+\eta(b, a))}{2}-\frac{1}{\eta(b, a)} \int_{a}^{a+\eta(b, a)} f(x) d x\right| \\
& \quad \leq \frac{\eta(b, a)}{4}\left[\frac{\left|f^{\prime}(a)\right|^{q}+\left|f^{\prime}(b)\right|^{q}}{2}\right]^{\frac{1}{q}} .
\end{aligned}
$$

Remark 6 If we take $\eta(b, a)=b-a$ in Theorem 23 , then the inequality reduces to inequality (1.12) from [5].

Remark 7 For $q=1$, (2.17) reduces to the inequality proved in Theorem 14. If $q=\frac{p}{p-1}$ $(p>1)$, we have $2^{p}>p+1$ for $p>1$ and, accordingly,

$$
\frac{1}{4}<\frac{1}{2(p+1)^{\frac{1}{p}}} .
$$

This reveals that inequality (2.17) is better than the one given by (1.17) in Theorem 15 from [29].

Now we give our results for prequasiinvex functions.

Theorem 24 Let $K \subseteq[0, \infty)$ be an open invex subset with respect to $\eta: K \times K \rightarrow \mathbb{R}$. Suppose that $f: K \rightarrow \mathbb{R}$ is a differentiable mapping on $K$ and $w:[a, a+\eta(b, a)] \rightarrow[0, \infty)$ is continuous and symmetric to $a+\frac{1}{2} \eta(b, a)$. If $\left|f^{\prime}\right|$ is prequasiinvex on $K$, then for every $a, b \in K$ with $\eta(b, a)>0$, we have the following inequality:

$$
\begin{aligned}
& \left|\frac{f(a)+f(a+\eta(b, a))}{2} \int_{a}^{a+\eta(b, a)} w(x) d x-\int_{a}^{a+\eta(b, a)} f(x) w(x) d x\right| \\
& \leq \frac{\eta(b, a)}{4}\left[\max \left\{\left|f^{\prime}(a)\right|,\left|f^{\prime}\left(a+\frac{1}{2} \eta(b, a)\right)\right|\right\}\right. \\
& \left.\quad+\max \left\{\left|f^{\prime}\left(a+\frac{1}{2} \eta(b, a)\right)\right|,\left|f^{\prime}(a+\eta(b, a))\right|\right\}\right] \int_{0}^{1} \int_{L^{\prime}(a, b, t)}^{u^{\prime}(a, b, t)} w(x) d x d t .
\end{aligned}
$$

Proof We continue inequality (2.8) in the proof of Theorem 21. Since $\left|f^{\prime}\right|$ is prequasiinvex on $K$, hence for every $t \in[0,1]$, we obtain

$$
\left|f^{\prime}\left(a+\left(\frac{1-t}{2}\right) \eta(b, a)\right)\right| \leq \max \left\{\left|f^{\prime}(a)\right|,\left|f^{\prime}\left(a+\frac{1}{2} \eta(b, a)\right)\right|\right\}
$$

and

$$
\left|f^{\prime}\left(a+\left(\frac{1+t}{2}\right) \eta(b, a)\right)\right| \leq \max \left\{\left|f^{\prime}\left(a+\frac{1}{2} \eta(b, a)\right)\right|,\left|f^{\prime}(a+\eta(b, a))\right|\right\} .
$$

A combination of (2.8), (2.19) and (2.20) gives the required inequality (2.18). 
Corollary 2 Suppose that all the conditions of Theorem 24 are satisfied. Moreover,

(1) if $\left|f^{\prime}\right|$ is non-decreasing, then the following inequality holds:

$$
\begin{aligned}
& \left|\frac{f(a)+f(a+\eta(b, a))}{2} \int_{a}^{a+\eta(b, a)} w(x) d x-\int_{a}^{a+\eta(b, a)} f(x) w(x) d x\right| \\
& \leq \frac{\eta(b, a)}{4}\left[\left|f^{\prime}\left(a+\frac{1}{2} \eta(b, a)\right)\right|+\left|f^{\prime}(a+\eta(b, a))\right|\right] \\
& \quad \times \int_{0}^{1} \int_{L^{\prime}(a, b, t)}^{U^{\prime}(a, b, t)} w(x) d x d t
\end{aligned}
$$

(2) if $\left|f^{\prime}\right|$ is non-increasing, then the following inequality holds:

$$
\begin{aligned}
& \left|\frac{f(a)+f(a+\eta(b, a))}{2} \int_{a}^{a+\eta(b, a)} w(x) d x-\int_{a}^{a+\eta(b, a)} f(x) w(x) d x\right| \\
& \quad \leq \frac{\eta(b, a)}{4}\left[\left|f^{\prime}(a)\right|+\left|f^{\prime}\left(a+\frac{1}{2} \eta(b, a)\right)\right|\right] \int_{0}^{1} \int_{L^{\prime}(a, b, t)}^{U^{\prime}(a, b, t)} w(x) d x d t .
\end{aligned}
$$

Remark 8 [31] If in Theorem 24 we take $w(x)=\frac{1}{\eta(b, a)}$ for all $x \in[a, a+\eta(b, a)]$ with $\eta(b, a)>$ 0 , then we have the following inequality:

$$
\begin{aligned}
& \left|\frac{f(a)+f(a+\eta(b, a))}{2}-\frac{1}{\eta(b, a)} \int_{a}^{a+\eta(b, a)} f(x) d x\right| \\
& \leq \frac{\eta(b, a)}{8}\left[\max \left\{\left|f^{\prime}(a)\right|,\left|f^{\prime}\left(a+\frac{1}{2} \eta(b, a)\right)\right|\right\}\right. \\
& \left.\quad+\max \left\{\left|f^{\prime}\left(a+\frac{1}{2} \eta(b, a)\right)\right|,\left|f^{\prime}(a+\eta(b, a))\right|\right\}\right] .
\end{aligned}
$$

Inequality (2.23) represents a new refinement of inequality (1.16) for prequasiinvex functions and hence for preinvex functions. Moreover,

(1) if $\left|f^{\prime}\right|$ is non-decreasing, then the following inequality holds:

$$
\begin{aligned}
& \left|\frac{f(a)+f(a+\eta(b, a))}{2}-\frac{1}{\eta(b, a)} \int_{a}^{a+\eta(b, a)} f(x) d x\right| \\
& \quad \leq \frac{\eta(b, a)}{8}\left[\left|f^{\prime}\left(a+\frac{1}{2} \eta(b, a)\right)\right|+\left|f^{\prime}(a+\eta(b, a))\right|\right] ;
\end{aligned}
$$

(2) if $\left|f^{\prime}\right|$ is non-increasing, then the following inequality holds:

$$
\begin{aligned}
& \left|\frac{f(a)+f(a+\eta(b, a))}{2}-\frac{1}{\eta(b, a)} \int_{a}^{a+\eta(b, a)} f(x) d x\right| \\
& \quad \leq \frac{\eta(b, a)}{8}\left[\left|f^{\prime}(a)\right|+\left|f^{\prime}\left(a+\frac{1}{2} \eta(b, a)\right)\right|\right] .
\end{aligned}
$$

Remark 9 If $\eta(b, a)=b-a$ in Theorem 24, then (2.18) reduces to inequality (1.13) established in Theorem 11 from [5], and inequalities (2.24) and (2.25) recapture the related inequalities given in the corollary of Theorem 11. 
Remark 10 If $\eta(b, a)=b-a$ in Remark 8, then (2.23) becomes inequality (1.8) of Theorem 6 from [2], and inequalities (2.24) and (2.25) recapture the related inequalities of the corollary of Theorem 6.

Theorem 25 Let $K \subseteq[0, \infty)$ be an open invex subset with respect to $\eta: K \times K \rightarrow \mathbb{R}$. Suppose that $f: K \rightarrow \mathbb{R}$ is a differentiable mapping on $K$ and $w:[a, a+\eta(b, a)] \rightarrow[0, \infty)$ is continuous and symmetric to $a+\frac{1}{2} \eta(b, a)$. If $\left|f^{\prime}\right|^{q}$ is prequasiinvex on $K$ for $q>1$, then for every $a, b \in K$ with $\eta(b, a)>0$, we have the following inequality:

$$
\begin{aligned}
& \left|\frac{f(a)+f(a+\eta(b, a))}{2} \int_{a}^{a+\eta(b, a)} w(x) d x-\int_{a}^{a+\eta(b, a)} f(x) w(x) d x\right| \\
& \leq \frac{\eta(b, a)}{4}\left(\int_{0}^{1}\left[\int_{L^{\prime}(a, b, t)}^{u^{\prime}(a, b, t)} w(x) d x\right]^{p} d t\right)^{\frac{1}{p}} \\
& \times\left[\left(\max \left\{\left|f^{\prime}(a)\right|^{q},\left|f^{\prime}\left(a+\frac{1}{2} \eta(b, a)\right)\right|^{q}\right\}\right)^{\frac{1}{q}}\right. \\
& \left.+\left(\max \left\{\left|f^{\prime}\left(a+\frac{1}{2} \eta(b, a)\right)\right|^{q},\left|f^{\prime}(a+\eta(b, a))\right|^{q}\right\}\right)^{\frac{1}{q}}\right],
\end{aligned}
$$

where $\frac{1}{p}+\frac{1}{q}=1$.

Proof We continue inequality (2.11) in the proof of Theorem 22. By the prequasiinvexity of $\left|f^{\prime}\right|^{q}$ on $K$ for $q>1$, we have, for every $t \in[0,1]$,

$$
\left|f^{\prime}\left(a+\left(\frac{1-t}{2}\right) \eta(b, a)\right)\right|^{q} \leq \max \left\{\left|f^{\prime}(a)\right|^{q},\left|f^{\prime}\left(a+\frac{1}{2} \eta(b, a)\right)\right|^{q}\right\}
$$

and

$$
\begin{aligned}
& \left|f^{\prime}\left(a+\left(\frac{1+t}{2}\right) \eta(b, a)\right)\right|^{q} \\
& \quad \leq \max \left\{\left|f^{\prime}\left(a+\frac{1}{2} \eta(b, a)\right)\right|^{q},\left|f^{\prime}(a+\eta(b, a))\right|^{q}\right\} .
\end{aligned}
$$

A combination of (2.11), (2.27) and (2.28) gives us the required inequality (2.26). This completes the proof of the theorem.

Corollary 3 Suppose that all the conditions of Theorem 25 are satisfied. Moreover,

(1) if $\left|f^{\prime}\right|^{q}$ is non-decreasing for $q>1$, then the following inequality holds:

$$
\begin{aligned}
& \left|\frac{f(a)+f(a+\eta(b, a))}{2} \int_{a}^{a+\eta(b, a)} w(x) d x-\int_{a}^{a+\eta(b, a)} f(x) w(x) d x\right| \\
& \leq \frac{\eta(b, a)}{4}\left[\left|f^{\prime}\left(a+\frac{1}{2} \eta(b, a)\right)\right|+\left|f^{\prime}(a+\eta(b, a))\right|\right] \\
& \quad \times\left(\int_{0}^{1}\left[\int_{L^{\prime}(a, b, t)}^{U^{\prime}(a, b, t)} w(x) d x\right]^{p} d t\right)^{\frac{1}{p}}
\end{aligned}
$$


(2) if $\left|f^{\prime}\right|^{q}$ is non-increasing for $q>1$, then the following inequality holds:

$$
\begin{aligned}
& \left|\frac{f(a)+f(a+\eta(b, a))}{2} \int_{a}^{a+\eta(b, a)} w(x) d x-\int_{a}^{a+\eta(b, a)} f(x) w(x) d x\right| \\
& \leq \frac{\eta(b, a)}{4}\left[\left|f^{\prime}(a)\right|+\left|f^{\prime}\left(a+\frac{1}{2} \eta(b, a)\right)\right|\right] \\
& \quad \times\left(\int_{0}^{1}\left[\int_{L^{\prime}(a, b, t)}^{U^{\prime}(a, b, t)} w(x) d x\right]^{p} d t\right)^{\frac{1}{p}},
\end{aligned}
$$

where $\frac{1}{p}+\frac{1}{q}=1$.

Remark 11 [31] If in Theorem 25 we take $w(x)=\frac{1}{\eta(b, a)}$ for all $x \in[a, a+\eta(b, a)]$ with $\eta(b, a)>0$, then we have the following inequality:

$$
\begin{aligned}
& \left|\frac{f(a)+f(a+\eta(b, a))}{2}-\frac{1}{\eta(b, a)} \int_{a}^{a+\eta(b, a)} f(x) d x\right| \\
& \leq \frac{\eta(b, a)}{4(p+1)^{\frac{1}{p}}}\left[\max \left\{\left|f^{\prime}(a)\right|,\left|f^{\prime}\left(a+\frac{1}{2} \eta(b, a)\right)\right|\right\}\right. \\
& \left.\quad+\max \left\{\left|f^{\prime}\left(a+\frac{1}{2} \eta(b, a)\right)\right|,\left|f^{\prime}(a+\eta(b, a))\right|\right\}\right] .
\end{aligned}
$$

Inequality (2.31) represents a new refinement of inequality (1.19) for prequasiinvex functions and hence for preinvex functions. Moreover,

(1) if $\left|f^{\prime}\right|$ is non-decreasing, then the following inequality holds:

$$
\begin{aligned}
& \left|\frac{f(a)+f(a+\eta(b, a))}{2}-\frac{1}{\eta(b, a)} \int_{a}^{a+\eta(b, a)} f(x) d x\right| \\
& \quad \leq \frac{\eta(b, a)}{4(p+1)^{\frac{1}{p}}}\left[\left|f^{\prime}\left(a+\frac{1}{2} \eta(b, a)\right)\right|+\left|f^{\prime}(a+\eta(b, a))\right|\right]
\end{aligned}
$$

(2) if $\left|f^{\prime}\right|$ is non-increasing, then the following inequality holds:

$$
\begin{aligned}
& \left|\frac{f(a)+f(a+\eta(b, a))}{2}-\frac{1}{\eta(b, a)} \int_{a}^{a+\eta(b, a)} f(x) d x\right| \\
& \leq \frac{\eta(b, a)}{4(p+1)^{\frac{1}{p}}}\left[\left|f^{\prime}(a)\right|+\left|f^{\prime}\left(a+\frac{1}{2} \eta(b, a)\right)\right|\right],
\end{aligned}
$$

where $\frac{1}{p}+\frac{1}{q}=1$.

Remark 12 If we take $\eta(b, a)=b-a$ in Remark 11, then (2.31) becomes inequality (1.9) of Theorem 7 from [2], and inequalities (2.32) and (2.33) become the related inequalities given in the corollary of Theorem 7 .

Theorem 26 Let $K \subseteq[0, \infty)$ be an open invex subset with respect to $\eta: K \times K \rightarrow \mathbb{R}$. Suppose that $f: K \rightarrow \mathbb{R}$ is a differentiable mapping on $K$ and $w:[a, a+\eta(b, a)] \rightarrow[0, \infty)$ is 
continuous and symmetric to $a+\frac{1}{2} \eta(b, a)$. If $\left|f^{\prime}\right|^{q}$ is prequasiinvex on $K$ for $q \geq 1$, then for every $a, b \in K$ with $\eta(b, a)>0$, we have the following inequality:

$$
\begin{aligned}
& \left|\frac{f(a)+f(a+\eta(b, a))}{2} \int_{a}^{a+\eta(b, a)} w(x) d x-\int_{a}^{a+\eta(b, a)} f(x) w(x) d x\right| \\
& \leq \frac{\eta(b, a)}{4}\left(\int_{0}^{1} \int_{L^{\prime}(a, b, t)}^{U^{\prime}(a, b, t)} w(x) d x d t\right)\left[\left(\max \left\{\left|f^{\prime}(a)\right|^{q},\left|f^{\prime}\left(a+\frac{1}{2} \eta(b, a)\right)\right|^{q}\right\}\right)^{\frac{1}{q}}\right. \\
& \left.\quad+\left(\max \left\{\left|f^{\prime}\left(a+\frac{1}{2} \eta(b, a)\right)\right|^{q},\left|f^{\prime}(a+\eta(b, a))\right|^{q}\right\}\right)^{\frac{1}{q}}\right] .
\end{aligned}
$$

Proof We continue inequality (2.15) in the proof of Theorem 23. By the prequasiinvexity of $\left|f^{\prime}\right|^{q}$ on $K$ for $q \geq 1$, we have, for every $t \in[0,1]$,

$$
\left|f^{\prime}\left(a+\left(\frac{1-t}{2}\right) \eta(b, a)\right)\right|^{q} \leq \max \left\{\left|f^{\prime}(a)\right|^{q},\left|f^{\prime}\left(a+\frac{1}{2} \eta(b, a)\right)\right|^{q}\right\}
$$

and

$$
\left|f^{\prime}\left(a+\left(\frac{1+t}{2}\right) \eta(b, a)\right)\right|^{q} \leq \max \left\{\left|f^{\prime}\left(a+\frac{1}{2} \eta(b, a)\right)\right|^{q},\left|f^{\prime}(a+\eta(b, a))\right|^{q}\right\} .
$$

A combination of (2.15), (2.35) and (2.36) gives us the required inequality (2.34). This completes the proof of the theorem.

Corollary 4 Suppose that all the conditions of Theorem 26 are satisfied. Moreover,

(1) if $\left|f^{\prime}\right|^{q}$ is non-decreasing for $q \geq 1$, then the following inequality holds:

$$
\begin{aligned}
& \left|\frac{f(a)+f(a+\eta(b, a))}{2} \int_{a}^{a+\eta(b, a)} w(x) d x-\int_{a}^{a+\eta(b, a)} f(x) w(x) d x\right| \\
& \leq \frac{\eta(b, a)}{4}\left(\int_{0}^{1} \int_{L^{\prime}(a, b, t)}^{u^{\prime}(a, b, t)} w(x) d x d t\right) \\
& \quad \times\left[\left|f^{\prime}\left(a+\frac{1}{2} \eta(b, a)\right)\right|+\left|f^{\prime}(a+\eta(b, a))\right|\right] ;
\end{aligned}
$$

(2) if $\left|f^{\prime}\right|^{q}$ is non-increasing for $q \geq 1$, then the following inequality holds:

$$
\begin{aligned}
& \left|\frac{f(a)+f(a+\eta(b, a))}{2} \int_{a}^{a+\eta(b, a)} w(x) d x-\int_{a}^{a+\eta(b, a)} f(x) w(x) d x\right| \\
& \quad \leq \frac{\eta(b, a)}{4}\left(\int_{0}^{1} \int_{L^{\prime}(a, b, t)}^{U^{\prime}(a, b, t)} w(x) d x d t\right)\left[\left|f^{\prime}(a)\right|+\left|f^{\prime}\left(a+\frac{1}{2} \eta(b, a)\right)\right|\right] .
\end{aligned}
$$

Remark 13 [31] If in Theorem 26 we take $w(x)=\frac{1}{\eta(b, a)}$ for all $x \in[a, a+\eta(b, a)]$ with $\eta(b, a)>0$, then we have inequality (1.22). Moreover,

(1) if $\left|f^{\prime}\right|^{q}$ is non-decreasing, then inequality (2.24) holds,

(2) if $\left|f^{\prime}\right|^{q}$ is non-increasing, then inequality (2.25) holds. 
Remark 14 If $\eta(b, a)=b-a$ in Theorem 26, then (2.34) reduces to inequality (1.14) established in Theorem 12 from [5], and inequalities (2.37) and (2.38) recapture the related inequalities established in the corollary of Theorem 12.

Remark 15 If $\eta(b, a)=b-a$ in Remark 13 , then (1.22) becomes inequality (1.10) of Theorem 8 from [2], and inequalities (2.24) and (2.25) recapture the related inequalities of the corollary of Theorem 8.

\section{Applications to special means}

In what follows we give certain generalizations of some notions for a positive valued function of a positive variable.

Definition 3 [34] A function $M: \mathbb{R}_{+}^{2} \rightarrow \mathbb{R}_{+}$is called a mean function if it has the following properties:

(1) Homogeneity: $M(a x, a y)=a M(x, y)$ for all $a>0$;

(2) Symmetry: $M(x, y)=M(y, x)$;

(3) Reflexivity: $M(x, x)=x$;

(4) Monotonicity: If $x \leq x^{\prime}$ and $y \leq y^{\prime}$, then $M(x, y) \leq M\left(x^{\prime}, y^{\prime}\right)$;

(5) Internality: $\min \{x, y\} \leq M(x, y) \leq \max \{x, y\}$.

We consider some means for arbitrary positive real numbers $\alpha, \beta$ (see, for instance, [34]).

(1) The arithmetic mean:

$$
A:=A(\alpha, \beta)=\frac{\alpha+\beta}{2} .
$$

(2) The geometric mean:

$$
G:=G(\alpha, \beta)=\sqrt{\alpha \beta} .
$$

(3) The harmonic mean:

$$
H:=H(\alpha, \beta)=\frac{2}{\frac{1}{\alpha}+\frac{1}{\beta}} .
$$

(4) The power mean:

$$
P_{r}:=P_{r}(\alpha, \beta)=\left(\frac{\alpha^{r}+\beta^{r}}{2}\right)^{\frac{1}{r}}, \quad r \geq 1 .
$$

(5) The identric mean:

$$
I:=I(\alpha, \beta)= \begin{cases}\frac{1}{e}\left(\frac{\beta^{\beta}}{\alpha^{\alpha}}\right), & \alpha \neq \beta, \\ \alpha, & \alpha=\beta .\end{cases}
$$

(6) The logarithmic mean:

$$
L:=L(\alpha, \beta)=\frac{\alpha-\beta}{\ln |\alpha|-\ln |\beta|}, \quad|\alpha| \neq|\beta| .
$$


(7) The generalized log-mean:

$$
L_{p}:=L_{p}(\alpha, \beta)=\left[\frac{\beta^{p+1}-\alpha^{p+1}}{(p+1)(\beta-\alpha)}\right], \quad \alpha \neq \beta, p \in \mathbb{R} \backslash\{-1,0\} .
$$

It is well known that $L_{p}$ is monotonic nondecreasing over $p \in \mathbb{R}$, with $L_{-1}:=L$ and $L_{0}:=I$. In particular, we have the inequality $H \leq G \leq L \leq I \leq A$.

Now, let $a$ and $b$ be positive real numbers such that $a<b$. Consider the function $M:=$ $M(a, b):[a, a+\eta(b, a)] \times[a, a+\eta(b, a)] \rightarrow \mathbb{R}^{+}$, which is one of the above mentioned means, therefore one can obtain variant inequalities for these means as follows.

Setting $\eta(b, a)=M(b, a)$ in (2.4), (2.10) and (2.14), one can obtain the following interesting inequalities involving means:

$$
\begin{aligned}
& \left|\frac{f(a)+f(a+M(a, b))}{2} \int_{a}^{a+M(a, b)} w(x) d x-\int_{a}^{a+M(a, b)} f(x) w(x) d x\right| \\
& \quad \leq \frac{M(a, b)}{4}\left[\left|f^{\prime}(a)\right|+\left|f^{\prime}(b)\right|\right] \int_{0}^{1} \int_{L^{\prime}(a, b, t)}^{U^{\prime}(a, b, t)} w(x) d x d t \\
& \left|\frac{f(a)+f(a+M(a, b))}{2} \int_{a}^{a+M(a, b)} w(x) d x-\int_{a}^{a+M(a, b)} f(x) w(x) d x\right| \\
& \quad \leq \frac{M(a, b)}{2}\left[\frac{\left|f^{\prime}(a)\right|^{q}+\left|f^{\prime}(b)\right|^{q}}{2}\right]^{\frac{1}{q}}\left(\int_{0}^{1}\left[\int_{L^{\prime}(a, b, t)}^{U^{\prime}(a, b, t)} w(x) d x\right]^{p} d t\right)^{\frac{1}{p}}
\end{aligned}
$$

for $q>1, \frac{1}{p}+\frac{1}{q}=1$ and

$$
\begin{aligned}
& \left|\frac{f(a)+f(a+M(a, b))}{2} \int_{a}^{a+M(a, b)} w(x) d x-\int_{a}^{a+M(a, b)} f(x) w(x) d x\right| \\
& \leq \frac{M(a, b)}{2}\left[\frac{\left|f^{\prime}(a)\right|^{q}+\left|f^{\prime}(b)\right|^{q}}{2}\right]^{\frac{1}{q}} \int_{0}^{1} \int_{L^{\prime}(a, b, t)}^{U^{\prime}(a, b, t)} w(x) d x d t
\end{aligned}
$$

for $q \geq 1$, where $U^{\prime}(a, b, t)=a+\left(\frac{1+t}{2}\right) M(a, b), L^{\prime}(a, b, t)=a+\left(\frac{1-t}{2}\right) M(a, b)$. Letting $M=$ $A, G, H, P_{r}, I, L, L_{p}$ in (3.1), (3.2) and (3.3), we can get the required inequalities for a different weight function $w(x)$, and the details are left to the interested reader.

\section{Competing interests}

The authors declare that they have no competing interests.

\section{Authors' contributions}

MAL and SSD both participated equally in writing all the results of the manuscript. All authors read and approved the final manuscript.

\section{Author details}

'School of Computational and Applied Mathematics, University of the Witwatersrand, Private Bag 3, Wits 2050,

Johannesburg, South Africa. ${ }^{2}$ School of Engineering and Science, Victoria University, P.O. Box 14428, Melbourne City, MC 8001, Australia.

Received: 7 August 2013 Accepted: 12 November 2013 Published: 05 Dec 2013

\section{References}

1. Pečarić, J, Proschan, F, Tong, YL: Convex Functions, Partial Ordering and Statistical Applications. Academic Press, New York (1991)

2. Alomari, M, Darus, M, Kirmaci, US: Refinements of Hadamard-type inequalities for quasi-convex functions with applications to trapezoidal formula and to special means. Comput. Math. Appl. 59, 225-232 (2010) 
3. Dragomir, SS, Agarwal, RP: Two inequalities for differentiable mappings and applications to special means of real numbers and trapezoidal formula. Appl. Math. Lett. 11(5), 91-95 (1998)

4. Dragomir, SS: Two mappings in connection to Hadamard's inequalities. J. Math. Anal. Appl. 167, 42-56 (1992)

5. Hwang, D-Y: Some inequalities for differentiable convex mapping with application to weighted trapezoidal formula and higher moments of random variables. Appl. Math. Comput. 217(23), 9598-9605 (2011)

6. Ion, DA: Some estimates on the Hermite-Hadamard inequality through quasi-convex functions. An. Univ. Craiova, Math. Comput. Sci. Ser. 34, 82-87 (2007)

7. Kırmacl, US: Inequalities for differentiable mappings and applications to special means of real numbers and to midpoint formula. Appl. Math. Comput. 147(1), 137-146 (2004)

8. Kırmacı, US, Özdemir, ME: On some inequalities for differentiable mappings and applications to special means of real numbers and to midpoint formula. Appl. Math. Comput. 153(2), 361-368 (2004)

9. Lee, KC, Tseng, KL: On a weighted generalization of Hadamard's inequality for G-convex functions. Tamsui Oxford Univ. J. Math. Sci. 16(1), 91-104 (2000)

10. Lupas, A: A generalization of Hadamard's inequality for convex functions. Univ. Beogr. Publ. Elektroteh. Fak., Ser. Mat. Fiz. 544-576, 115-121 (1976)

11. Pearce, CEM, Pečarić, J: Inequalities for differentiable mappings with application to special means and quadrature formulae. Appl. Math. Lett. 13(2), 51-55 (2000)

12. Qi, F, Wei, Z-L, Yang, Q: Generalizations and refinements of Hermite-Hadamard's inequality. Rocky Mt. J. Math. 35, 235-251 (2005)

13. Sarıkaya, MZ, Aktan, N: On the generalization some integral inequalities and their applications. Math. Comput. Model. 54(9-10), 2175-2182 (2011)

14. Sarikaya, MZ, Avci, M, Kavurmaci, H: On some inequalities of Hermite-Hadamard type for convex functions. ICMS International Conference on Mathematical Science. AIP Conf. Proc. 1309, 852 (2010)

15. Sarikaya, MZ: O new Hermite-Hadamard Fejér type integral inequalities. Stud. Univ. Babeş-Bolyai, Math. 57(3), 377-386 (2012)

16. Saglam, A, Sarikaya, MZ, Yıldırım, H: Some new inequalities of Hermite-Hadamard's type. Kyungpook Math. J. 50 399-410 (2010)

17. Wang, C-L, Wang, X-H: On an extension of Hadamard inequality for convex functions. Chin. Ann. Math. 3, 567-570 (1982)

18. Wu, S-H: On the weighted generalization of the Hermite-Hadamard inequality and its applications. Rocky Mt. J. Math. 39(5), 1741-1749 (2009)

19. Hanson, MA: On sufficiency of the Kuhn-Tucker conditions. J. Math. Anal. Appl. 80, 545-550 (1981)

20. Ben-Israel, A, Mond, B: What is invexity? J. Aust. Math. Soc. Ser. B 28(1), 1-9 (1986)

21. Pini, R: Invexity and generalized convexity. Optimization 22, 513-525 (1991)

22. Noor, MA: Invex equilibrium problems. J. Math. Anal. Appl. 302, 463-475 (2005)

23. Noor, MA: Variational-like inequalities. Optimization 30, 323-330 (1994)

24. Yang, XM, Li, D: On properties of preinvex functions. J. Math. Anal. Appl. 256, 229-241 (2001)

25. Weir, T, Mond, B: Preinvex functions in multiple objective optimization. J. Math. Anal. Appl. 136, 29-38 (1998)

26. Noor, MA: On Hadamard integral inequalities involving two log-preinvex functions. J. Inequal. Pure Appl. Math. 8(3), 1-14 (2007)

27. Yang, XM, Yang, XQ, Teo, KL: Characterizations and applications of prequasi-invex functions. J. Optim. Theory Appl. $110,645-668(2001)$

28. Noor, MA: Hermite-Hadamard integral inequalities for log-preinvex functions. Preprint

29. Barani, A, Ghazanfari, AG, Dragomir, SS: Hermite-Hadamard inequality for functions whose derivatives absolute values are preinvex. RGMIA Res. Rep. Collect. 14, Article ID 64 (2011)

30. Barani, A, Ghazanfari, AG, Dragomir, SS: Hermite-Hadamard inequality through prequasiinvex functions. RGMIA Res. Rep. Collect. 14, Article ID 48 (2011)

31. Latif, MA: Some inequalities for differentiable prequasiinvex functions with applications. Konuralp J. Math. 1(2), 17-29 (2013)

32. Sarikaya, MZ, Bozkurt, H, Alp, N: On Hermite-Hadamard type integral inequalities for preinvex and log-preinvex functions. arXiv:1203.4759v1

33. Mohan, SR, Neogy, SK: On invex sets and preinvex functions. J. Math. Anal. Appl. 189, $901-908$ (1995)

34. Bullen, PS: Handbook of Means and Their Inequalities. Kluwer Academic, Dordrecht (2003)

$10.1186 / 1029-242 X-2013-575$

Cite this article as: Latif and Dragomir: Some weighted integral inequalities for differentiable preinvex and

prequasiinvex functions with applications. Journal of Inequalities and Applications 2013, 2013:575 